\title{
Large-Eddy Simulation of a microburst
}

\author{
V. Anabor ${ }^{1}$, U. Rizza ${ }^{1,2}$, E. L. Nascimento ${ }^{1}$, and G. A. Degrazia ${ }^{1}$ \\ ${ }^{1}$ Universidade Federal de Santa Maria, Departamento de Física, Santa Maria, RS, Brazil \\ ${ }^{2}$ Institute for Atmospheric Sciences and Climate, ISAC-CNR, Italy
}

Received: 15 September 2010 - Published in Atmos. Chem. Phys. Discuss.: 20 October 2010

Revised: 16 February 2011 - Accepted: 23 August 2011 - Published: 9 September 2011

\begin{abstract}
The three-dimensional structure and evolution of an isolated and stationary microburst are simulated using a time-dependent, high resolution Large-Eddy-Simulation (LES) model. The microburst is initiated by specifying a simplified cooling source at the top of the domain around $2 \mathrm{~km}$ a.g.l. that leads to a strong downdraft. Surface winds of the order of $30 \mathrm{~m} \mathrm{~s}^{-1}$ were obtained over a region of $500 \mathrm{~m}$ radius around the central point of the impinging downdraft, with the simulated microburst lasting for a few minutes. These characteristic length and time scales are consistent with results obtained from numerical simulations of microbursts using cloud-resolving models. The simulated flow replicated some of the principal features of microbursts observed by Doppler radars: in particular, the horizontal spread of strong surface winds and a ring vortex at the leading edge of the cold outflow. In addition to the primary surface outflow, the simulation also generated a secondary surge of strong winds that appears to represent a pulsation in the microburst evolution.

These results highlight the capability of LES to reproduce complex phenomena like microbursts, indicating the potential usage of LES models to represent atmospheric phenomena of time and space scales between the convective scale and the microscale. These include short-lived convectivelygenerated damaging winds.
\end{abstract}

\section{Introduction}

During the life cycle of a thunderstorm, complex circulations are developed. In its developing stage the storm structure is dominated by low-level convergence and deep and intense updrafts that transport moisture and warm air from the

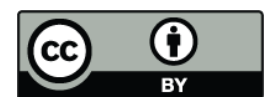

Correspondence to: U. Rizza

(u.rizza@isac.cnr.it) environmental boundary layer to the storm. As precipitation forms, negative buoyancy is generated due to evaporation of raindrops in contact with entrained air (or the melting of solid hydrometeors). At this stage stronger downdrafts are formed while updrafts are weakened. On some occasions the cooled air becomes so negatively buoyant that intense downdrafts are generated inducing strong winds at ground level. This physical mechanism can lead to the formation of downbursts (Fujita, 1985).

Fujita defines a downburst as a short-lived strong downdraft that induces a highly divergent outburst. These damaging winds can be either straight or curved. A microburst is defined as a small-scale downburst, where the horizontal extent of the damaging winds is less than $4 \mathrm{~km}$. Despite its small horizontal scale, an intense microburst can induce damaging winds as strong as $75 \mathrm{~m} \mathrm{~s}^{-1}\left(270 \mathrm{~km} \mathrm{~h}^{-1}\right)$. For such reasons microbursts can be specially dangerous to aircraft during takeoff and landing manoeuvres due to the intense wind shear accompanying them (Elmore et al., 1986). Microbursts have been the subject of several observational and numerical studies since the pioneering works of Fujita (1985). Field studies such as NIMROD (Fujita 1979), JAWS (McCarthy et al., 1982), FLOWS (Wolfson et al., 1987) and BAMEX (Davis et al., 2004) have sampled a large number of microbursts with single and multiple Doppler radar. These field studies allowed a better understanding of the physical properties of microbursts, and helped foster the development of numerical models that simulate microburst-producing storms.

Generally, numerical models employed to study microbursts fall into two main categories: sub-cloud models and cloud-resolving microburst models (Orf and Anderson, 1999). In the former category some sort of forcing - representing the bulk effect of microphysical processes that generate negative buoyancy in a storm - is usually imposed in the upper portion of the model domain in order to initiate the microburst (Anderson et al., 1992; Orf et al., 1996); in the

Published by Copernicus Publications on behalf of the European Geosciences Union. 
latter category the full life cycle of the microburst-producing storm is simulated (Hjelmfelt et al., 1989; Proctor, 1988, 1989; Straka and Anderson, 1993). In an attempt to model the microburst phenomenon, Srivastava $(1985,1987)$ used simple one-dimensional time dependent models of strong downdrafts forced by evaporative cooling and melting precipitation. Orf and Anderson (1999) used a $k-\varepsilon$ model to investigate the effects of travelling microbursts in unidirectional sheared environments. In the context of numerical modelling of microbursts with engineering applications some recent studies include that of Nicholls et al. (1993), which performed a 2-D very high resolution simulation of a microburst on a building model. Kim and Hangan (2007) used the CFD software Fluent 6.0 (FLUENT, 2001) in a 2-D setup to investigate the macro-scale flow dynamics of an impinging jet. Sengupta et al. (2001) performed both experimental and numerical simulations of microburst winds, while Mason et al. (2009) developed a dry 2-D non-hydrostatic model implemented within the CFD commercial code ANSYS CFX11 (ANSYS, 2007).

Sub-cloud numerical simulations of microbursts not including cloud microphysics nor large scale atmospheric processes can be performed at very high resolution, allowing the analysis of detailed structure of the near-ground flow dynamics, where the strongest wind speeds are usually detected in microbursts. The thermal forcing for these simulations is usually parameterized using a spatio-temporal variable function that is decoupled from microphysics and moisture advection (Orf et al., 1996).

In the present study the microburst dynamics is investigated using a Large-Eddy-Simulation (LES) model that has been widely employed by the scientific community to study the properties of planetary boundary layer (PBL) flows. LES is a well-established technique to study the 3-D turbulent characteristics of PBL, as vastly documented in the literature (Lesieur and Métais, 1996). Nevertheless, the number of LES studies addressing sub-cloud modelling of microbursts is still scarce mainly due to the required high computational cost. More recently, the availability of less expensive platforms for parallel computing has made the LES strategy more appealing and not as prohibitive as in the past.

The aim of this study is to evaluate the ability of LES in reproducing the intense downward wind currents associated with a microburst. This will be accomplished with idealised conditions typical of sub-cloud modelling in which the cooling forcing that generates the microburst is parameterized following Orf et al. (1996).

\section{Mechanisms driving microbursts}

A microburst event is originated by microphysical and thermodynamic processes in a convective cloud (Mahoney and Rodi, 1987). These processes are explored by Srivastava $(1985,1987)$ using a simple one-dimensional time- dependent model of downdrafts driven by evaporation and melting of hydrometeors. The downdraft intensity increases as the environmental lapse rate becomes less stable and as the raindrop size decreases (Srivastava, 1985). When the environmental temperature profile approaches the dry-adiabatic lapse rate, even very light precipitation can produce intense downdrafts. As stable stratification increases, progressively higher cloud water content (liquid and ice) is needed to produce intense downdrafts (Srivastava, 1987). An environment displaying both a sub-cloud temperature profile that is near dry-adiabatic and a high concentration of small hydrometeors undergoing melting or evaporation is highly conducive to the generation of microbursts (Wakimoto, 1985, 2001; Wakimoto et al, 1994).

Atlas et al. (2004) analysed a unique set of Doppler and polarimetric radar observations of a microburst-producing storm during Tropical Rainfall Measuring Mission (TRMM) Large-Scale Biosphere-Atmosphere (LBA) field experiment. The results closely matched the initial conditions and results of Srivastava $(1985,1987)$ that suggest that only modest size hail in large concentration melting aloft is needed to produce wet microbursts. The narrower the distribution of hail particle sizes, the more confined will be the layer of melting and negative buoyancy, and the more intense the microburst.

The downdraft acceleration can be schematically represented by the vertical component of the equation of motion (Bluestein, 2007).

$$
\frac{D w}{D t}=-\frac{1}{\rho_{0}} \frac{\partial p^{*}}{\partial z}+B\left(T, r_{\mathrm{v}}, r_{1}, r_{\mathrm{i}}\right)
$$

where $w$ is the vertical velocity, $p *$ is the pressure perturbation from the basic state, and $B$ is the buoyancy term which depends on temperature $(T)$, water vapour mixing ratio $\left(r_{\mathrm{v}}\right)$ and hydrometeor loading $\left(r_{1}, r_{i}\right)$, as follows (Bluestein 2007):

$B=\left[g T^{\prime}\left(1+1.609 r_{\mathrm{v}}-r_{1}-r_{\mathrm{i}}\right)\right] / T_{0}$

where $T^{\prime}$ is the temperature deviation from the basic state, $T_{0}$ is the basic state temperature, $r_{1}$ is the rainwater mixing ratio and $r_{\mathrm{i}}$ is the ice mixing ratio.

While the pressure gradient force plays an important role in the dynamics of severe thunderstorms, the buoyancy term is recognized as the most important forcing mechanism for microbursts (Orville et al, 1989; Wakimoto, 1985, 2001; Wakimoto et al, 1994).

\section{Model description}

To perform the sub-cloud modelling of a microburst, we adapted the Large-Eddy-Simulation code of Moeng (1984). The code version chosen is the dry one, such that no microphysical parameterisation is needed. The complete derivation of the resolved scale equations can be found in Moeng (1984) and Sullivan et al. (1994). 
Only the equations that are relevant to the understanding of the microburst dynamics are discussed here. In our simulations the negative buoyancy that drives the microburst formation is parameterised by imposing an additional source term $Q(\mathrm{x}, \mathrm{y}, \mathrm{z} ; t)$ in the equation for the virtual potential temperature, as follows:

$$
\frac{\partial \bar{\theta}}{\partial t}=-\bar{u}_{j} \frac{\partial \bar{\theta}}{\partial x_{j}}-\frac{\partial \tau_{\theta j}}{\partial x_{j}}+Q(\mathrm{x}, \mathrm{y}, \mathrm{z} ; t)
$$

where $\bar{\theta}$ is the resolved virtual potential temperature field, $\left(\bar{u}_{1}, \bar{u}_{2}, \bar{u}_{3}\right) \equiv(\bar{u}, \bar{v}, \bar{w})$ is the resolved velocity field and $\tau_{\theta j}$ is the tensor that represents the subgrid closure for the temperature field. The boundary conditions in the horizontal were periodic, the upper boundary was specified as a frictionless rigid lid with zero mass, momentum, heat and subgrid kinetic energy flux, and the bottom boundary employed a noslip condition with a prescribed roughness length.

The use of a cooling function, in the context of subcloud modelling, avoids the use of explicit microphysics routines, which are computationally very expensive. Furthermore the shape, duration and amplitude parameters of the cooling function may be easily chosen providing an easier interpretation of the modelling results.

\section{Methodology}

The main driving mechanism for the downburst is given by the buoyancy term in Eq. (1) that represents physical processes such as cooling due to melting and evaporation of hydrometeors.

In the LES configuration a cooling function parameterising the bulk effect of these processes is placed at the top the domain, near $2 \mathrm{~km}$.

In the sub-cloud LES model used here the spatial-temporal cooling function is specified following Orf et al. (1996), that is:

$$
Q(x, y, z ; t)= \begin{cases}g(t) \cos ^{2}(\pi R) & \text { for } R<1 / 2 \\ 0 & \text { for } R>1 / 2\end{cases}
$$

where $R$ is the normalised distance from the centre of cooling, given by

$$
R=\sqrt{\left(\frac{x-x_{f}}{M_{\mathrm{x}}}\right)^{2}+\left(\frac{y-y_{f}}{M_{\mathrm{y}}}\right)^{2}+\left(\frac{z-z_{f}}{M_{\mathrm{z}}}\right)^{2}}
$$

where $\left(x_{f}, y_{f}, z_{f}\right)$ is the location of the center of the forcing function and $\left(M_{\mathrm{x}}, M_{\mathrm{y}}, M_{\mathrm{z}}\right)$ is its horizontal/vertical extension (Table 1).

The variable $g(t)$ is the time modulation of the magnitude of the forcing function that follows Orf et al. (1996), being described by:

$$
\begin{cases}g(t)=-\cos ^{2}\left[\pi\left(\frac{t-120}{2 \tau}\right)\right] & 0 \leq t<120 \\ g(t)=-1 & 120 \leq t \leq 720 \\ g(t)=-\cos ^{2}\left[\pi\left(\frac{t-720}{2 \tau}\right)\right] & 720<t \leq 840\end{cases}
$$

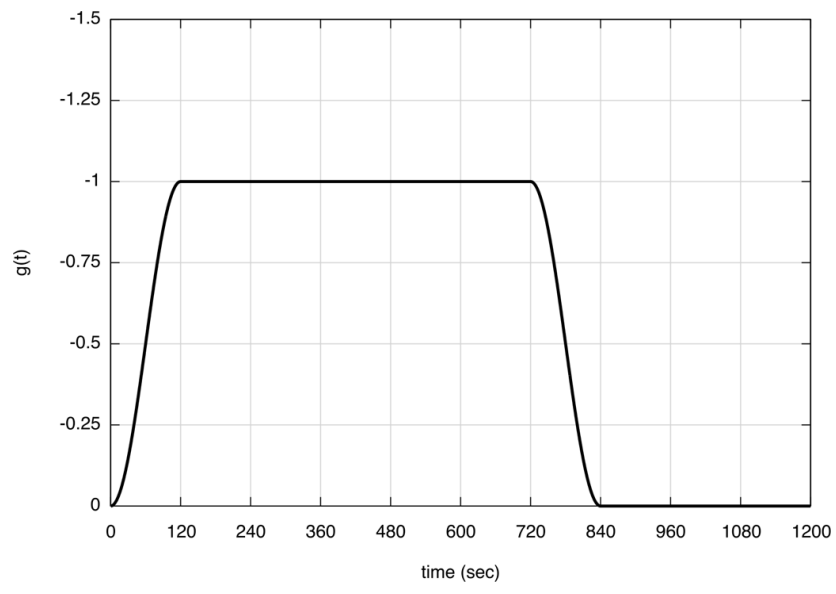

Fig. 1. Temporal modulation of the maximum intensity of the cooling function.

Table 1. Position $\left(\mathrm{x}_{f}, \mathrm{y}_{f}, \mathrm{z}_{f}\right)$ and extension $\left(M_{\mathrm{x}}, M_{\mathrm{y}}, M_{\mathrm{y}}\right)$ of the forcing function and its Cooling Rate.

\begin{tabular}{rrr}
\hline$\left(\mathrm{x}_{f}, \mathrm{y}_{f}, \mathrm{z}_{f}\right)$ & $\left(M_{\mathrm{x}}, M_{\mathrm{y}}, M_{\mathrm{y}}\right)$ & $\mathrm{CR}$ \\
\hline $\mathrm{km}$ & $\mathrm{km}$ & $\mathrm{Ks}^{-1}$ \\
$(5,5,2)$ & $(2,2,1.8)$ & 0.08 \\
\hline
\end{tabular}

where $\tau=120 \mathrm{~s}$. This means that it ramps to a maximum value after $2 \mathrm{~min}$, after which a constant value is kept for $10 \mathrm{~min}$, followed by a gradual decrease in the last $2 \mathrm{~min}$ as depicted in Fig. 1. An intense source as employed in Mason et al. (2009) has been used here, with a maximum cooling rate of $-0.08 \mathrm{~K} \mathrm{~s}^{-1}$ to produce a more intense downburst event. In fact, Orville et al. (1989) found cooling rates due to evaporation of raindrops approaching $10^{-1} \mathrm{~K} \mathrm{~s}^{-1}$ for the strongest microburst cases.

Dry microbursts typically occur within a deep dryadiabatic sub-cloud layer (or mixed layer) that might extend up to $2 \mathrm{~km}$ a.g.1. or higher (Wakimoto, 2001). Because the mixed layer is nearly in a dry-neutral condition throughout most of its depth, the specification of a neutral PBL is our choice for the background environment for the idealized numerical simulation of a microburst. In this context, LES is an adequate numerical tool to simulate a neutral PBL (Andren et al., 1994). By varying the geostrophic wind and the surface heat flux, different turbulent regimes can be generated with LES. A prototype of a neutral PBL may be obtained by first generating a convective PBL by imposing an upward heat flux, followed by a gradual weakening of the heat flux, allowing a quick spin-up to a steady shear flow (Moeng and Sullivan, 1994).

In the present work, simulations were performed in a $10 \times 10 \mathrm{~km}$ horizontal rectangular domain that is $2 \mathrm{~km}$ deep with $128^{3}$ grid points. The convective simulation started 

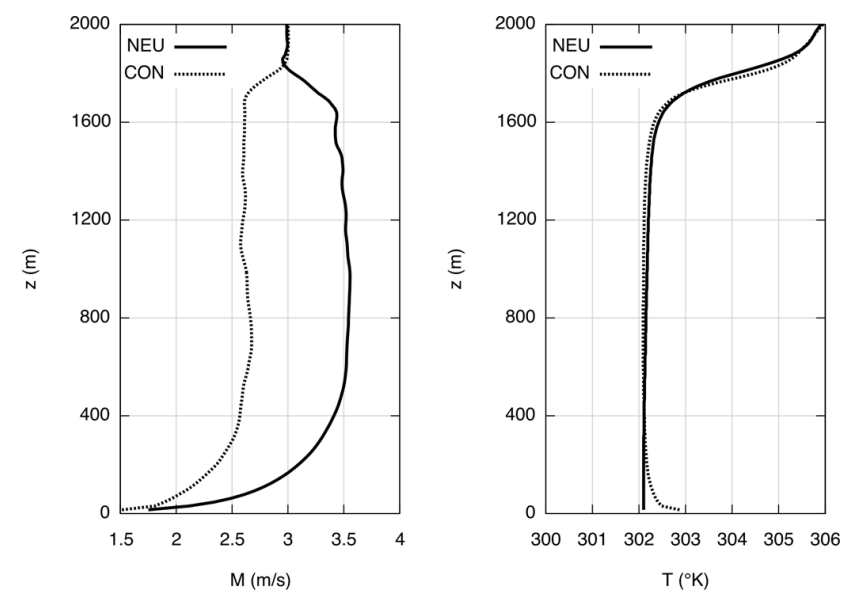

Fig. 2. Magnitude of the mean wind speed profiles (left) and potential temperature (right) in the convective (dotted line) and neutral (continuous line) PBL.

from a barotropic condition, that is, with the geostrophic wind constant throughout the numerical domain. Internal parameters such as the extension of domain, grid size, geostrophic wind, kinematic surface heat flux $\left(Q_{*}\right)$, and initial capping inversion height $\left(z_{\mathrm{i}}\right)_{0}$ are summarised in Table 2. After the quasi-stationary regime is reached by the convective boundary layer (CBL) the heat flux was turned off and a neutral PBL was simulated for the following 6 h. In Fig. 2 are shown the mean wind and temperature profile snapshots taken at the end of the convective simulation $(\mathrm{CON})$ and at the end of neutral simulation (NEU). It should be noticed that a profile with vertical wind shear is attained as a consequence of the surface frictional stresses. Meanwhile the profile of constant potential temperature, typical of a neutral PBL conditions, is evident in Fig. 2.

The cooling forcing function is activated exactly at the end of the NEU simulation. According to the imposed time modulation of the forcing function (Fig. 1) the forcing lasted for $14 \mathrm{~min}$ and covered an area of (2.2) $\mathrm{km}$ in the upper region of the simulation domain.

\section{Results and discussion}

\subsection{Qualitative comparisons}

In the case of an isolated static microburst, the main features to note are the downdraft core, wall jet and the roll vortices (Orf et al., 1996). The term "wall jet" is usually employed in fluid dynamics to illustrate the typology of shallow flow that occurs when a cold fluid impinges a flat surface at high speed (Hjelmfelt, 1988).

The cooling function applied at the top of the domain generates a downdraft with vertical velocity reaching approximately $20 \mathrm{~m} \mathrm{~s}^{-1}$. Just after the impact with the ground a cold pool is generated around the impact point. The
Table 2. Internal parameters of the LES domain.

\begin{tabular}{rrrrr}
\hline$\left(N_{\mathrm{x}}, N_{\mathrm{y}}, N_{\mathrm{Z}}\right)$ & $\left(L_{\mathrm{x}}, L_{\mathrm{y}}, L_{\mathrm{z}}\right)$ & $Q_{*}$ & $\left(U_{\mathrm{g}}, V_{\mathrm{g}}\right)$ & $\left(z_{i}\right)_{0}$ \\
\hline & & $\left(\mathrm{ms} \mathrm{K}^{-1}\right)$ & $\mathrm{m} \mathrm{s}^{-1}$ & $\mathrm{~m}$ \\
$128^{3}$ & $(10,10,2)$ & 0.25 & $(3,0)$ & 1500 \\
\hline
\end{tabular}

hydrostatically-induced pressure gradient force across the pressure dome transfers the momentum associated with the impinging downdraft to a cold outflow current. This leads to the development of a divergent wall jet, corresponding to the highest wind speeds generated in this simulation.

At the leading edge of the wall jet a vortex ring circulation is generated. The origin of the vorticity in such feature is the strong horizontal gradient in buoyancy that exists along the edge of the outflow (Proctor, 1988). The wall jet, as a negatively buoyant downdraft, maintains and expands the vortex ring circulation. The sequence of Fig. 3a-e depicts the magnitude of surface winds speeds at the first vertical level of the LES grid, respectively 3, 5, 6, 8 and 10 min into the simulation. The general picture that may be understood examining Fig. 3a-e is the following: around the impact point, just a few seconds after the cold plume impinges on the surface, a strong surge in the wind field is developed (Fig. 3a, b). The highest surface speeds are generated during this stage for the reasons explained above.

The expansion of this surge is maintained by the strong horizontal pressure gradient that is hydrostatically associated with the drop in temperature across the leading edge of the cold plume. As this surge spreads outwards, the effect of turbulent mixing gradually reduces the temperature gradient at the leading edge weakening the horizontal pressure gradient (Fig. 3c). This process evolves in a time scale of the order of 1 min.

In the meantime, the cold downdraft continues impinging on the surface forming a secondary surge in the wind field (Fig. 3d). The passage of the first cold surge modifies the low levels of the PBL reducing the horizontal pressure gradient force within the outflow, which results in a considerably weaker radial acceleration of the second surge (Fig. 3e). Accordingly, the horizontal buoyancy gradient is also weakened inside the pool of cold air, such that the generation of horizontal vorticity due to baroclinic effect (Proctor, 1988) is reduced, avoiding the formation of a new vortex ring associated with the secondary surge. This second surge in the wind field, despite not characterizing a true additional burst, appears to represent a manifestation of a pulsation in the microburst behaviour, which has been detected in radar velocity fields (see Fig. 8 of Hjelmfelt, 1988) and studied in Proctor (1993). Further studies are necessary to better understand the basic dynamics behind such behaviour.

The reproduction of a neutral PBL that is typical of the microburst environment has as effect the presence of a predominant longitudinal mean wind $\left(\sim 3 \mathrm{~m} \mathrm{~s}^{-1}\right)$ and a weak 

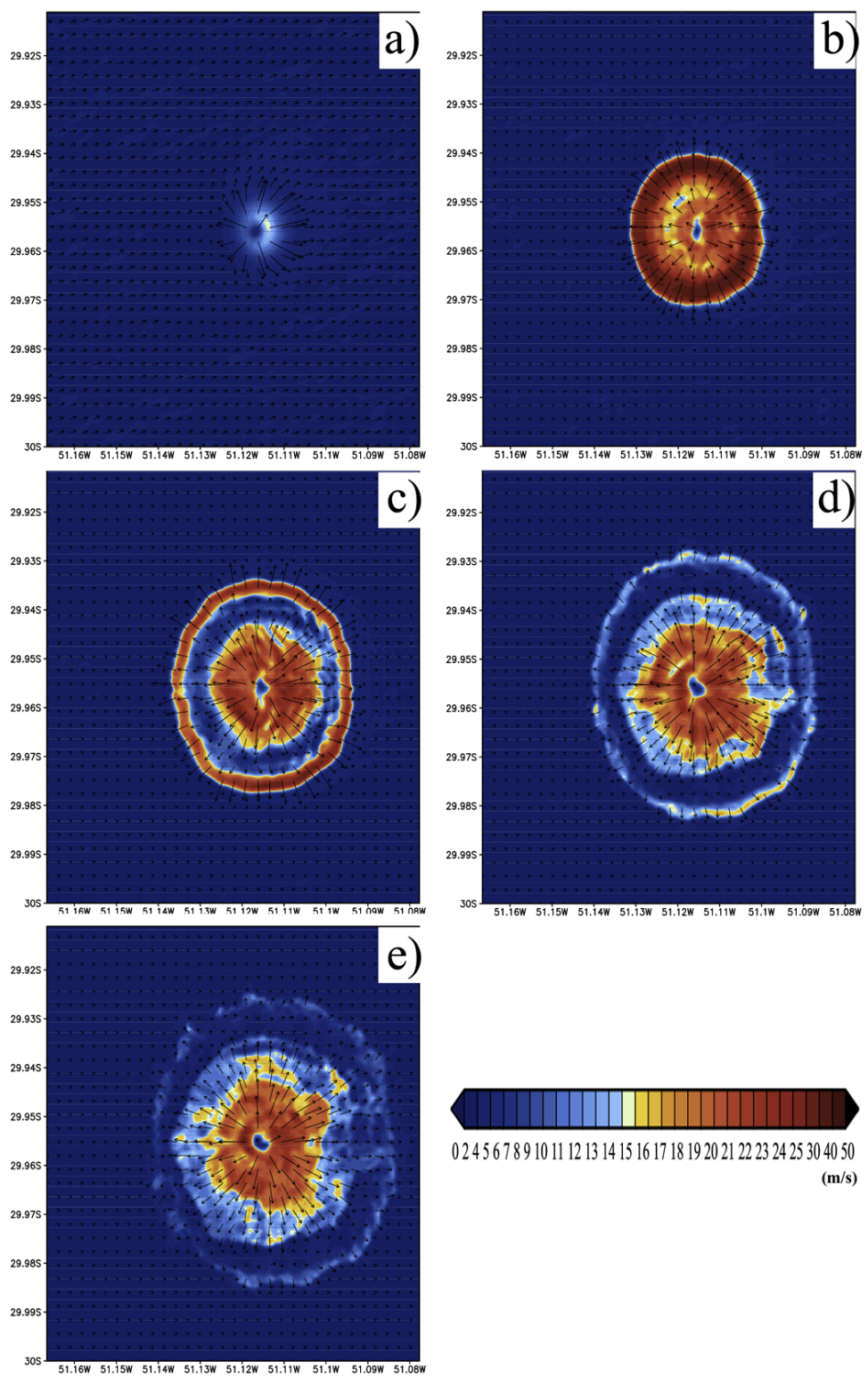

e)

0245678910111213141516171819202122232425304050

$(\mathrm{m} / \mathrm{s})$

Fig. 3. Magnitude of surface winds $\left(\mathrm{m} \mathrm{s}^{-1}\right.$ ) at the first vertical level of the LES grid, respectively (a) 3 , (b) 5, (c) 6, (d) 8 and (e) 10 min after the start of cooling.

lateral component $\left(\sim 0.3 \mathrm{~m} \mathrm{~s}^{-1}\right)$. During the microburst development it is possible to identify latitudinal symmetry and longitudinal asymmetry along the vortex ring (Fig. 3c).

This asymmetry is the result of the predominant wind $(u$ component) interaction with the vertical velocity in the microburst plume. Figure 4a shows a mean flow channelling into the cold microburst. Figure $4 \mathrm{~b}$ depicts the evidence of the vortex ring development along the upwind side of the microburst as a result of the interaction between the mean flow and the microburst surface upstream currents. Along the downwind side of the microburst the vortex ring is generated a few minutes later, since the microburst outflow has the same direction of the mean flow (Fig. 4b,c).
Figure 5 shows the vertical distribution of the maximum radial velocity. The radial velocity is calculated from the centre of the numerical domain, at each radial distance with a bilinear interpolation of the resolved horizontal components $(\bar{u}, \bar{v})$ in the eight closest points of the LES grid. The radial wind evolution shown in this figure suggests that nonhydrostatic pressure perturbations are present, accelerating the surface winds before the maximum radial wind velocity region in the vertical wind profile reaches the ground. This feature is evident in Fig. 5 from 135 to $174 \mathrm{~s}$. Notice that the maximum velocity of the impinging jet, after $84 \mathrm{~s}$ is located at about $1000 \mathrm{~m}$ a.g.l. with a speed of $8 \mathrm{~m} \mathrm{~s}^{-1}$. This is possibly caused by the horizontal divergence induced by the 


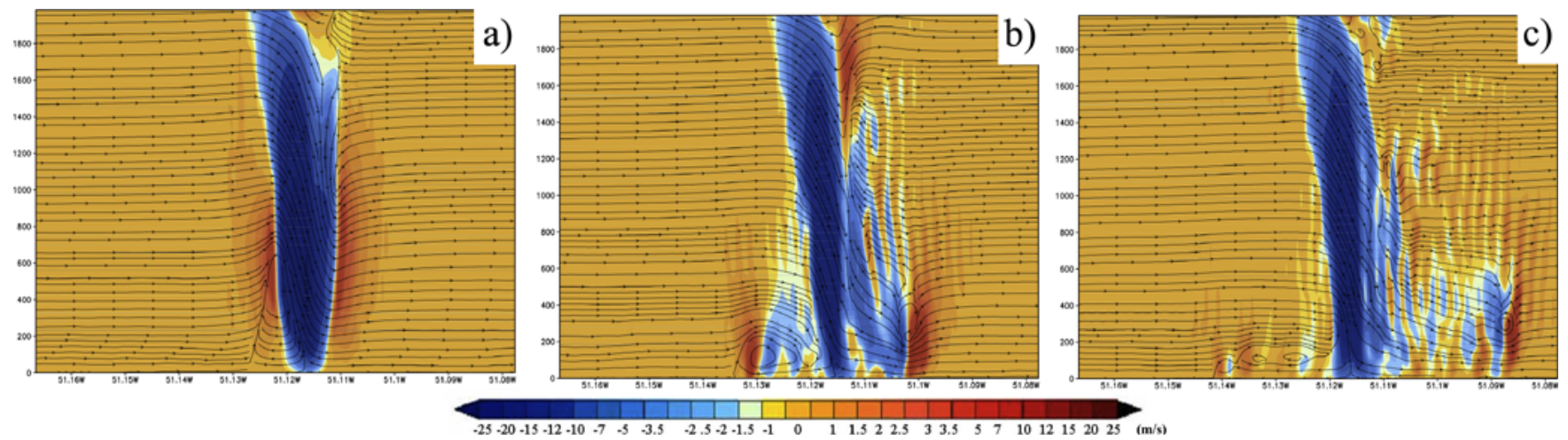

Fig. 4. Vertical velocity $\left(\mathrm{m} \mathrm{s}^{-1}\right)$ in a $(\mathrm{x}-\mathrm{z})$ section of the LES domain, respectively (a) 3 , (b) 5 and (c) 8 min after the start of cooling.

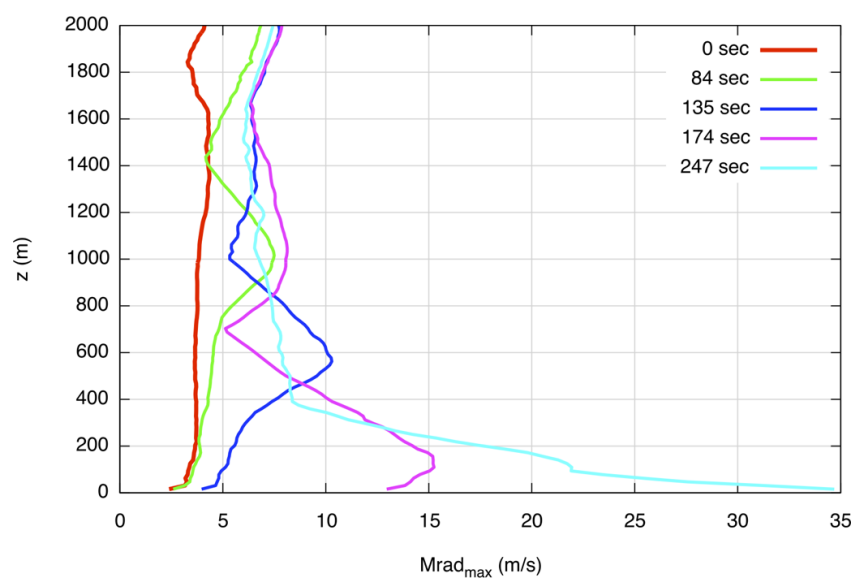

Fig. 5. Vertical distribution of the maximum of the radial velocity. Times are taken after the start of cooling (red line).

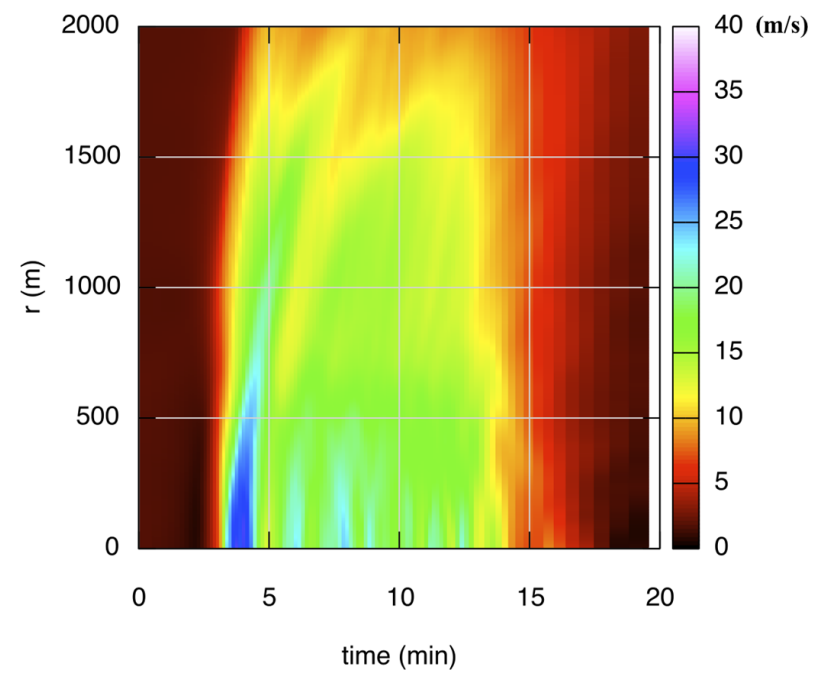

Fig. 6. Time-radial distance plot of the wind field magnitude $\left(\mathrm{m} \mathrm{s}^{-1}\right)$ at the first vertical level of the LES domain.

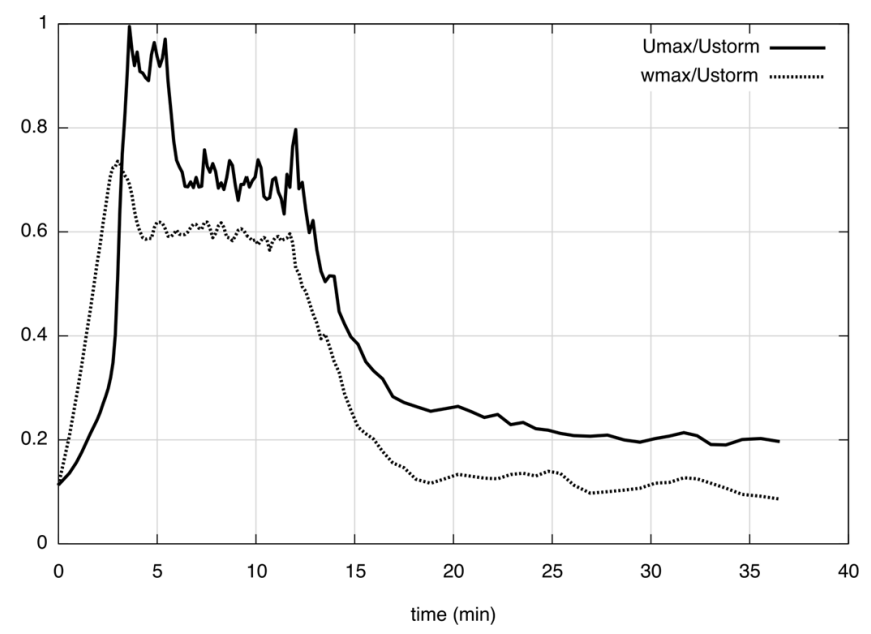

Fig. 7. Temporal variation of the normalized maximum storm event wind speed $\left(U_{\max }\right)$ and the maximum downdraft velocity $\left(w_{\max }\right)$.

passage of the cold downdraft at that level. After $135 \mathrm{~s}$ the maximum $\left(10 \mathrm{~m} \mathrm{~s}^{-1}\right)$ is located about $600 \mathrm{~m}$ a.g.l.; after $174 \mathrm{~s}$ it is located at $150 \mathrm{~m}$ a.g.l. and the speed is close to $15 \mathrm{~m} \mathrm{~s}^{-1}$. After impact, the surface wind speed increases rapidly reaching $35 \mathrm{~m} \mathrm{~s}^{-1}$ in almost one minute. This picture shows that the radial velocity is growing and displacing downward following the edge of the cold plume. This is also in agreement with the classic "picture" describing a microburst as characterized by strong localized downflow and an outburst of strong winds near the surface. The time-radial distance plot (Fig. 6) shows the surface wind speeds $\left(M=\left(U^{2}+V^{2}\right)^{1 / 2}\right)$ and spatial structure of the magnitude of the wind field at the first vertical level of the LES grid. A remarkable feature is evident: the first surge forms $4-5$ min after the start of cooling with highest speeds $\left(\sim 30 \mathrm{~m} \mathrm{~s}^{-1}\right)$ occurring in the first $500 \mathrm{~m}$, and decreasing radially from the microburst centre. The vortex ring is present at the leading edge of the surge expansion and travels for about $2000 \mathrm{~m}$ in less than $5 \mathrm{~min}$. The secondary surge appears two min after the formation of 
the first surge $(\sim 6 \mathrm{~min})$ with lower speeds $\left(\sim 20 \mathrm{~m} \mathrm{~s}^{-1}\right)$ and smaller expansion $(\sim 1500 \mathrm{~m})$.

There is a reduction of the horizontal pressure gradient after the passage of the vortex ring. Therefore, smaller wind speeds are observed during the secondary surge expansion. During the radial expansion is possible to notice the kinetic energy dissipation (Fig. 6).

A quasi-periodic $(\sim 2 \mathrm{~min})$ oscillation is present close to the microburst center (first $500 \mathrm{~m}$ ) as observed in the timeradial plot of the wind speed.

Doppler radar observations show that a microburst is characterized when the downward current reaches the ground generating a strong divergence at its center, such that an accelerated outburst of surface winds can be observed (Wakimoto, 2001). This simulation closely reproduces the Doppler Radar observations and highlights the LES capability to reproduce complex thermal and dynamical phenomena like microbursts, jet streaks and other events involving high velocities.

\subsection{Quantitative comparison}

The maximum simulated storm event wind speed $U_{\text {storm }}$ (Mason et al. 2009) was $38.4 \mathrm{~m} \mathrm{~s}^{-1}$ at the first LES vertical level $(\approx 16 \mathrm{~m})$ four minutes after the start of cooling. The maximum downdraft velocity was $W_{\max }=-28.6 \mathrm{~ms}^{-1}$ at almost $500 \mathrm{~m}$ a.g.1. The value of $U_{\text {storm }} / W_{\max } \approx 1.4$ observed for this simulation is in close agreement with similar values (1.6) obtained from simulations of Proctor (1989) and Mason et al. (2009). Figure 7 shows the time series of $U_{\max }$ and $W_{\max }$ which represent respectively the maximum values of $U$ and $W$ observed anywhere in the domain in the given time steps. The simulated wind speed was normalised with respect to $U_{\text {storm }}$, to allow the analysis of the maximum speed as compared to with references to $U_{\text {storm }}$. The interpretation of this figure is straightforward if analysed again in terms of the primary and secondary surge expansion mentioned earlier. The first "plateau" of $U_{\text {max }} / U_{\text {storm }}$ depicted between 3 and $6 \mathrm{~min}$ is related to the expansion of the primary surge, while the second plateau (between 6 and 13 min) appears to be related with the formation of the secondary surge with a lower value of $U_{\max } / U_{\text {storm }} \approx 0.65$. The maximum value of vertical velocity is around $0.75 U_{\text {storm }}$ at elevated region, located $500 \mathrm{~m}$ a.g.l. found just before the downdraft impinges the surface. After that, it keeps a constant value of $0.60 U_{\text {storm }}$ for almost 10 minutes before decaying toward the dissipation stage.

The last analysis performed is showed in Fig. 8a, where vertical distribution of the normalized maximum velocity is indicated.

The wind speed profile is normalised with respect to the peak mean wind speed, and height is normalised with respect to the height where the velocity is equal to half its maximum value (Wood et al., 2001). Semi-empirical results based on full scale data (Wood et al., 2001), experimental data (Did-
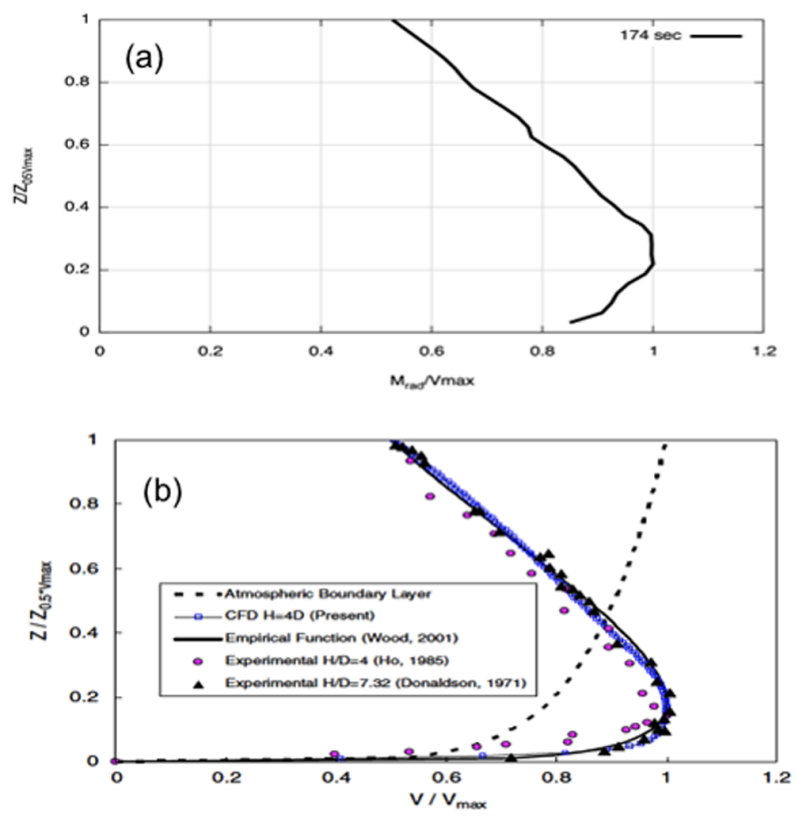

Fig. 8. Vertical distribution of the normalized maximum velocity in self-similar coordinate, (a) present simulation, (b) semi-empirical results (reprinted with permission of Journal of Wind Engineering and Industrial Aerodynamics).

den and Ho, 1985; Donaldson and Snedeker, 1971), are reported in Fig. 8b (Kim and Hangan, 2005). The comparison of Fig. 8a and b highlights the good agreement between the present simulation and experimental data.

\section{Conclusions}

An isolated and stationary microburst is simulated using a 3-D time-dependent, high resolution Large-Eddy Simulation model with the goal of studying the evolution and the dynamical structure of the microburst. The microburst-producing downdraft is initiated by specifying a simplified cooling source at the top of the boundary in place of a computationally expensive full cloud model. The simulated microburst displayed a vortex ring feature that propagates outward following the leading edge of the cold outflow. The peak horizontal wind speed occurred in association with the expansion of the first surge. The simulated time scale for this damaging wind $\left(30 \mathrm{~m} \mathrm{~s}^{-1}\right)$ is of the order of few minutes with a spatial scale enclosing a region with $500 \mathrm{~m}$ radius around the impact point. The simulated flow reproduces some of the main features of microbursts observed by Doppler Radar, in particular a very strong surge in the wind field, and the formation of the vortex ring at the leading edge of the cold outflow. In addition, the simulation suggests the presence of a second surge of strong winds trailing the first outburst which may be a manifestation of a pulsating behaviour of the microburst which has been documented by Hjelmfelt (1988) and studied 
by Proctor (1993). In all of these phases the role of the surface pressure gradient is evidenced and discussed. Additionally, the modelled maximum storm velocity is comparable to that obtained from full-cloud models (Proctor, 1989) and experimental data (Kim and Hangan, 2005).

This evidences the capability of LES in reproducing complex phenomena like a microburst. In fact, LES contains in its basic equations all physical information needed to create the basics mechanism for describing the microburst dynamics: the accelerated downward cold plume, the primary surge expansion due to the horizontal pressure gradient and its interaction with environment in order to generate the vortex ring expansion.

These results indicate the potential of LES for utilization in atmospheric phenomena situated below the storm scale and above the microscale, which generally involves high velocities in a short time scale.

Acknowledgements. The authors are gratefully indebted to CNPq (Conselho Nacional de Desenvolvimento Cientifico e Tecnológico, Brasil) and CNR (Consiglio Nazionale delle Ricerche, Italia) for the financial support of this work.

Edited by: T. J. Dunkerton

\section{References}

Anderson, J. R., Orf, L. G. and Straka, J. M.: A 3-D model system for simulating thunderstorm microburst outflows, Meteorol. Atmos. Phys., 49, 125-131, 1992.

Andren, A., Brown, A. R., Graf, J., Mason, J., Moeng, C. -H., Nieuwstadt F. T. M., and Schumann, U.: Large-eddy-simulation of a neutrally stratified boundary layer: a comparison of four computer codes, Q. J. Roy. Meteor. Soc., 120, 1457-1484, 1994.

ANSYS: ANSYS CFX User's Manual, available at: http://www. ansys.com/Products/cfx, 2007.

Atlas, D., Ulbrich, C. W., and Willams, C. R.: Physical origin of a wet microburst: observations and theory, J. Atmos. Sci., 61, 1186-1195, 2004.

Bluestein, H. B.: Advances in applications of the physics of fluids to severe weather systems, Reg. Prog. Phys., 70, 1259-1323, 2007.

Davis, C., Atkins, N., Bartels, D., Bosart, L., Coniglio, M., Bryan, G., Cotton, W., Dowell, D., Jewett, B., Johns, R., Jorgensen, D., Knievel, J., Knupp, K., Lee, W. -C., McFarquhar, G., Moore, J., Przybylinski, R., Rauber, R., Smull, B., Trapp, R., Trier, S., Wakimoto, R., Weisman, M., and Ziegler, C.: The Bow Echo and MCV experiment, Bull. Amer. Meteorol. Soc., 85, 1075-1093, 2004.

Didden, N. and Ho, C. M.: Unsteady separation in a boundary layer produced by an impinging jet, J. Fluid Mech., 160, 235-256, 1985.

Donaldson, C. D. and Snedeker, R. S.: A study of free jet impingement, Part 1. Mean properties of free and impinging jets, J. Fluid Mech., 45, 281-319, 1971.

Elmore, K. L., MacCarthy, J., Frost, W. and Chang, H. P.: A high resolution spatial and temporal multiple Doppler analysis for a microburst and its application to aircraft flight simulation, J. Climate Appl. Meteorol., 25, 1398-1425, 1986.

FLUENT: 6.0 User's Guide 2001, 1-4, Fluent Inc., Lebanon, December 2001.

Fujita, T. T.: Objectives, operation, and results of Project NIMROD, Preprints, 11th Conf. on Severe Local Storms, Kansas City, MO, American Meteorological Society, 259-266, 1979.

Fujita, T. T.: The downburst, microburst and macroburst, sattellite and mesometeorology research project (SMRP), Research Paper 210, Dept. of Geophysical Sciences, Univ. of Chicago, USA, (NTIS PB-148880), 1985.

Hjelmfelt, M. R.: Structure and life cycle of microburst outflows observed in Colorado, J. Climate Appl. Meteor., 27, 900-927, 1988.

Hjelmfelt, M. R., Orville, H. D., Roberts, R. D., Chen, J. P., and Kopp F. J.: Observational and numerical study of a microburst line-producing storm, J. Atmos. Sci., 46, 2731-2743, 1989.

Kim, J. and Hangan, H.: Numerical simulations of impinging jets with application to downbursts, J. Wind Eng. Ind. Aerod., 95, 279-298, 2007.

Kim, J., Ho, E., and Hangan, H.: Downburst induced dynamic responses of tall buildings. In: Proceedings of the 10th Americas Conference on Wind Engineering (10 ACWE), Baton Rouge, LA, USA, 2005.

Lesieur, M. and Métais, O.: New trends in large-eddy simulation, Annu. Rev. Fluid Mech., 28, 45-82, 1996.

Mason, S. M., Wood, G. S. D. F., and Fletcher D. F.: Numerical simulation of downburst winds, J. Wind Eng. Ind. Aerod., 97, 523-539, 2009.

Mahoney, W. P. and Rodi, A. R: Aircraft measurements on microburst development from hydrometeor evaporation, J. Atmos. Sci., 44, 3037-3051, 1987.

McCarthy, J., Wilson, J. W., and Fujita, T. T.: The Joint Airport Weather Studies Project, B. Am. Meteorol. Soc., 63, 15-22, 1982.

Moeng, C.-H.: A Large-eddy-simulation model for the study of planetary boundary-layer turbulence, J. Atmos. Sci., 41, 20522062, 1984.

Moeng, C.-H. and Sullivan, P. P.: A Comparison of shear and buoyancy driven planetary boundary layer flows, J. Atmos. Sci., 51, 999-1021, 1994.

Nicholls, M., Pielke, R., and Meroney, R.: Large eddy simulation of microburst winds flowing around a building, J. Wind. Eng. Ind. Aerod., 46-47, 229-237, 1993.

Orf, L. G. and Anderson, J. R.: A numerical study of traveling microbursts, Mon. Weather Rev., 127, 1244-1257, 1999.

Orf, L. G., Anderson, J. R., and Straka, J. M.: A three-dimensional numerical analysis of colliding microburst outflow dynamics, J. Atmos. Sci., 53, 2490-2511, 1996.

Orville, H. D., Farley, R. D., and Chi, Y.-C., Kopp, F. J.: The primary cloud physics mechanisms of microburst formation, Atmos. Res., 24, 343-357, 1989.

Proctor, F. H.: Numerical simulations of an isolated microburst. Part I: Dynamics and structure, J. Atmos. Sci., 45, 3137-3160, 1988.

Proctor, F. H: Numerical simulations of an isolated microburst, Part II: Sensitivity experiments, J. Atmos. Sci., 46, 2143-2165, 1989.

Proctor, F. H.: Case study of a low-reflectivity pulsating microburst: numerical simulation of the Denver, 8 July 2009, storm. In: Proceedings of the 17th Conference on Severe 
Local Storms, American Meteorological Society, St. Louis/USA, 677-680, 1993.

Sengupta, A., Sarkar, P. P., and Rajagopalan, G.: Numerical and physical simulation of thunderstorm downdraft winds and their effects on buildings, in: First American Conference on Wind Engineering, Clemson, USA, 2001.

Srivastava, R. C.: A simple model of evaporatively driven downdrafts: application to microburst downdraft, J. Atmos. Sci., 42, 1004-1023, 1985.

Srivastava, R. C.: A model of intense downdrafts driven by melting and evaporation of precipitation, J. Atmos. Sci., 44, 1752-1773, 1987.

Straka, J. M. and Anderson, J. R.: The numerical simulations of microburst-producing thunderstorms: some results from storms observed during the COHMEX experiment, J. Atmos. Sci., 50, 1329-1348, 1993.

Sullivan, P. P., McWilliams, J. C., and Moeng, C.-H.: A subgridscale model for large-eddy simulation of planetary boundarylayer flows, Bound.-Lay. Meteorol., 71, 247-276, 1994.
Wakimoto, R. M.: Forecasting microburst activity over the High Plains, Mon. Weather Rev., 113, 1131-1143, 1985.

Wakimoto, R. M.: Convectively-driven high wind events in: Severe Convective Storms, edited by: Doswell, C. A. III, American Meteorological Society, Meteorological Monographs, 28(50), 255298, 2001.

Wakimoto, R. M., Kessinger, C. J. and Kingsmill, D. E.: Kinematic, thermodynamic, and visual structure of low-reflectivity microbursts, Mon. Weather Rev., 122, 72-92, 1994.

Wood, G. S., Kwok, K. C. S., Motteram, N. A., and Fletcher, D. F. Physical and numerical modelling of thunderstorm downbursts, J. Wind Eng. Ind. Aerod., 89, 535-552, 2001.

Wolfson, M. M., Distefano, J. T., and Forman, B. E.: The FLOWS Automatic Weather Station Network in Operation. MIT Lincoln Laboratory, Project Report ATC-134, FAA Report DOT-FAAPM-85/27, 1987. 